\title{
Performance Analysis of Smart Energy Monitoring Systems in Real-time
}

\author{
R. Govindarajan \\ Department of Electrical Engineering \\ SCSVMV University \\ Kanchipuram, India \\ govind@kanchiuniv.ac.in
}

\author{
S. Meikandasivam \\ School of Electrical Engineering \\ Vellore Institute of Technology \\ Vellore, India \\ meikandasivam.s@vit.ac.in
}

\author{
D. Vijayakumar \\ School of Electrical Engineering \\ Vellore Institute of Technology \\ Vellore, India \\ vijayakumar.d@vit.ac.in
}

\begin{abstract}
The use of smart energy monitoring systems in buildings offers significant potential for energy saving. The introduction of a new kind of energy monitoring system is essential in the consumer-side in order to meet the demands of energy conservation. In this paper, four energy monitoring systems based on advanced wireless technology are proposed. The design and implementation of the smart real-time energy monitoring systems is analyzed with respect to their performance. The proposed systems utilize advanced wireless technologies like the Zigbee module, Internet of Things (IoT), Android Mobile Apps, and cloud computing for interfacing the information between the meters and the consumer end. A digital power meter is installed in the main panel, interfaced with a communication gateway and parameters such as voltage, current, power, power factor, and the harmonics of household appliances are measured in real-time. These systems provide power consumption details to the consumer and allow interacting with them by giving instant data such as live power tracking, identification of abnormal patterns of energy usage, energy bill estimation, and energy usage information.
\end{abstract}

Keywords-ZigBee; Internet of Things; GSM communication; cloud computing; smart grid

\section{INTRODUCTION}

Smart Grid (SG) gives the energy industry the opportunity to move into a new era of more reliable and efficient systems. The Smart Energy Monitoring System (SEMS) is one of the most important devices used in a Smart Grid. It is an advanced energy metering unit that obtains the information from consumers and provides information to the utility company and system operators. Several sensors and control devices that are supported by dedicated communication infrastructure are utilized in the SEMS. In addition, SEMS uses a smart meter developed using system's communication technologies [1]. The SEMS is expected to work in the following way: It collects data from nodes, establishes two-way communication, analyzes data, and supports controlling based on the analysis. Initially, the data are collected from the appliances and are recorded at regular time intervals. The collected data are stored in the memory and data prioritization is done. The command signals are received from the utility center from which the smart meter plans for load scheduling and controlling based on consumer behavior. Automatic electricity billing system can also be implemented in SEMS, because the system supports bidirectional information flow and is deployed at the end user premises to collect the data of electrical usage from all the appliances. The data from the individual smart meters are collected by local data aggregators and then the information is passed to the utility center. Similarly, the utility center also can send some commands or signals to the smart meters at end user premises whenever required. The SEMS should communicate the data to the utility center in a highly reliable and secured way. The system collects the data from local smart meters and sends them to the utility companies using different wired and wireless technologies involving HAN, NAN and WAN. The data communication in HAN is carried out by using Power Line Communication (PLC), Radio Frequency (RF), Zigbee, WiFi. The data communication in NAN involves copper or optical fiber, Wi-Fi, general packet radio service (GPRS) and WiMax. The data communication using WAN includes optical fibers, cellular networks, and satellite connections [2].

With the advancement in the field of computer and wireless communication technology, a wide range of possibilities is available for the design and implementation of SEMS in future smart homes [3]. Computer and wireless communication technology is used to integrate a number of sensor devices, actuators, and computing devices which in turn have great potential to contribute to energy efficiency in everyday usage. Energy efficiency is becoming increasingly important in industry and in the residential sector [4]. However, due to the complexity and diversity of computing devices, integrating energy monitoring resources is still in its infancy [5]. Adding advanced devices into the energy metering environment is a very challenging task. After deciding which particular device to integrate, the smart metering developer must determine how to configure it and interface with it [6]. The smart energy monitoring environment makes use of a wireless communication platform to integrate various devices. These devices should communicate with each other independently to provide information to the consumers [7]. Manufacturers of wireless interfacing devices use many different communication modules. Therefore, the integration of household appliances and consumer electronics is not straightforward at any layer of the sensor network platform. Additionally, apart from the technical difficulties of the integration, due to the complexity of the systems, users are often unable to fully understand their 
systems [8]. To fully take advantage of smart energy monitoring technologies, any energy monitoring system should transparently communicate with all the household energy meters and make available all important information to the users with respect to saving energy and user convenience [9]. Keeping these in mind, SEMSs using wireless communication module and an intelligent gateway circuit are proposed. The proposed systems continually record and update the electricity consumption data in order to provide real-time information. Users can easily monitor and control their power consumption of household appliances in order to save energy. To enable the feasibility of the proposed systems, Zigbee transceivers and a GSM communication gateway are implemented. In order to find the most suitable energy monitoring system for consumers, the following systems are designed and implemented using various wireless technologies and the experimental results are discussed.

- SEMS using the ZigBee module.

- SEMS using IoT.

- SEMS using Android apps.

- SEMS using cloud computing.

\section{IMPEMENTATION}

\section{A. Design and Implementation in a ZigBee Module}

The ZigBee Module (IEEE 802.15.4) is a promising wireless communication protocol for low rate communication and home automation. Conventional wired and wireless communication interfaces like Ethernet, IEEE 1394, Wi-Fi, PLC, and Bluetooth can be used for the home network. Wireless network interfaces are preferred to wired communication, because they are easy to deploy and install. Additionally, ZigBee has a higher maximum number of cell nodes than other protocols. For example, Bluetooth has only 8 nodes as maximum number of cell nodes, while ZigBee can have more than 65000 nodes [10]. The proposed ZigBee-based energy monitoring system includes an Energy Surveillance Monitor (ESM) that has a Zigbee transceiver and multiple Energy Surveillance Devices (ESDs). The ESM is used for displaying the collected data to the user and the ESDSs perform functions like energy consumption measurement, adaptive setting of standby power-off level and Zigbee communication. The main function and performance requirements of the system are [11]:

- Adaptive standby power level setting.

- Standby power-off control.

- Power consumption measurement.

- Zigbee communication.

ZigBee is one of the most economical and reliable modules which also consumes less power for monitoring processes. It covers a wireless range of 10-100 meters. An energy meter can be interfaced with the ZigBee transmitter module and a laptop or computer is used for data acquisition [12]. In this system, an embedded $\mathrm{C}$ programming is developed for the interfacing process and a power metering IC is used to collect the data from the energy meters. The collected data will be communicated to the ZigBee Module using the Arduino Controller. Finally, the measured electrical parameters will be received by the ZigBee transceiver. This way, the data are presented in the form of an energy report, are viewable to the consumers, and can be accessed from a PC or a laptop. The hardware implemented for an energy monitoring system using the ZigBee Module is shown in Figure 1. In this system, the digital meter is interfaced with the ZigBee transmitter board and a PC is interfaced with the ZigBee receiver board. There is no other wired connection between the energy meter and the PC. The system is implemented for wireless energy monitoring. This wireless setup can cover a maximum distance of $100 \mathrm{~m}$ and the configuration is as per IEEE standard [12, 13]. The system collects energy data from the digital energy meter using an Arduino controller through a power amplifier. The collected data will be indicated in the Arduino board by the flickering LED. The signal status is also updated by it [3]. The The Power Amplifier IC will act as a data splitter. It is used to provide the data to the controller without any jungle values of the energy meter. The measured energy parameters will be sent to the PC and displayed as a text report as shown in Figure 2.

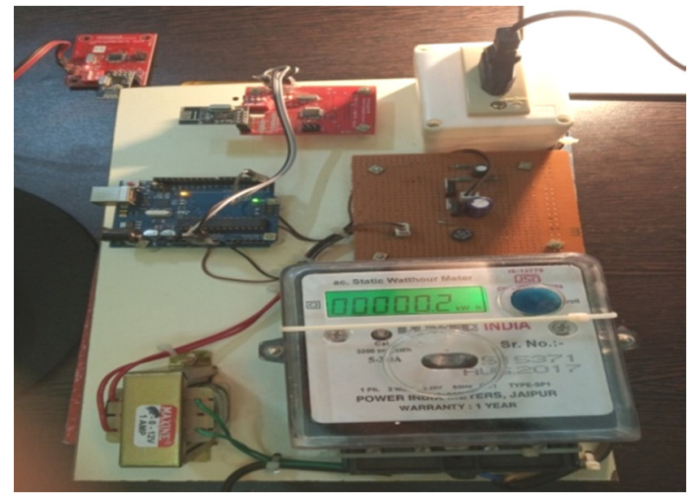

Fig. 1. Hardware implementation of the Zigbee-based energy monitoring system

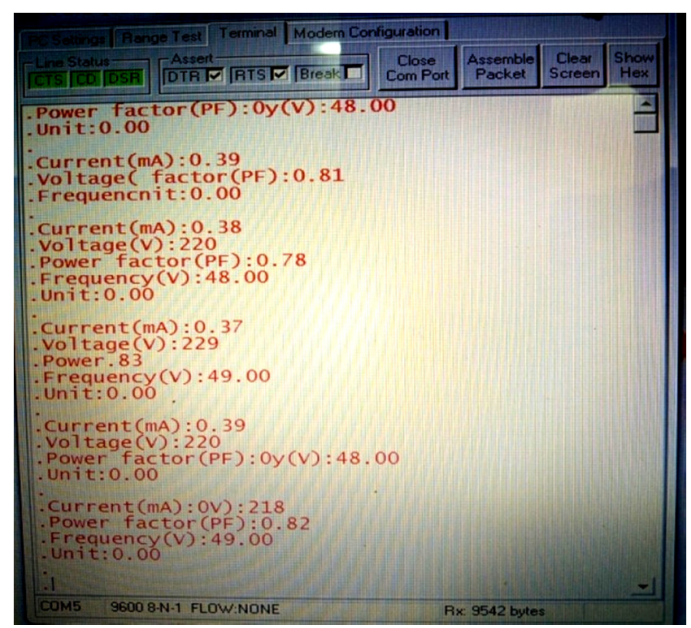

Fig. 2. Energy meter values display format on computer screen

For this system interfacing, the coding was developed with the Arduino processor by Embedded C [14]. The system 
provides the information to users with a delay time of $15 \mathrm{~s}$ and the data communication speed between the energy meter and the $\mathrm{PC}$ is $250 \mathrm{kbps}$.

\section{B. Design and Implementation Using IoT}

An IoT (IEEE 1451-99) based SEMS allows the devices to remotely sense using the existing internet infrastructure and also develop the interfacing process between the transmitter end (energy meter) and the receiver end. In addition, it reduces human participation in process handling. This system is implemented with customized embedded programming between IoT and the digital meter through the Arduino controller $[14,15]$. In the proposed system, the consumer can maintain energy consumption by knowing the energy usage from time to time. This method not only provides two way communication between the utility and the consumer but also it provides other functions, e.g. when the consumer fails to pay the electricity bill, the energy supply will be cut down from the utility side and once the bill is paid the energy supply can be reconnected automatically without human intervention. IoT is a major part of the communication between the energy meter and the web server. The sensor collects data from load and the energy meter, which data are then sent to the Arduino through UART communication. The controller then performs the operation logically and automatically using MQTT protocol. These data are then stored on the cloud and they can be monitored any time. The stored data are displayed on the web, where the user and the utility company can monitor them and perform suitable actions. The data are updated with the real time status of the connected device and are sent to the controller to take the appropriate action. The designed system will provide data transferring speed from the monitoring unit to server of $2 \mathrm{mbps}$ with a time delay of $2 \mathrm{~s}$. The monthly bill will be sent to the consumer in the form of an energy report with IP address accessing mode. Figure 3 demonstrates the operation of a developed IoT based energy monitoring system. The system consists of a user-interface dashboard and it was developed in HTML, which is used to view data as an energy monitoring report [16]. The system is designed to find the minimum period of data transmission delay time with high communication speed. The normal digital energy meter was connected with an interfacing unit [17]. The interfacing unit consists of the Arduino, a splitter circuit, a data amplifier circuit, and the IoT module. The interfacing unit collects the energy parameters from the energy meter and sends them to the local server through IoT. In this system, the splitter circuit will split the data into voltage, current, and power and send them to the power amplifier circuit. The amplifier circuit and the Arduino processor will store the values and make a live report as shown in Figure 4. The measured energy parameters were collected with an interfacing unit and then were sent to the local server through a $\mathrm{Wi}-\mathrm{Fi}$ router. Before that, the energy report is prepared as an HTML file in the background and all the fields are developed on consumer basic requirements. Based on the programming, the data will appear in content menu or field [18]. Each consumer can view its energy consumption report by accessing its already given IP address [20]. Hence, this proposed system achieves fast communication with very small data transmission delay time.

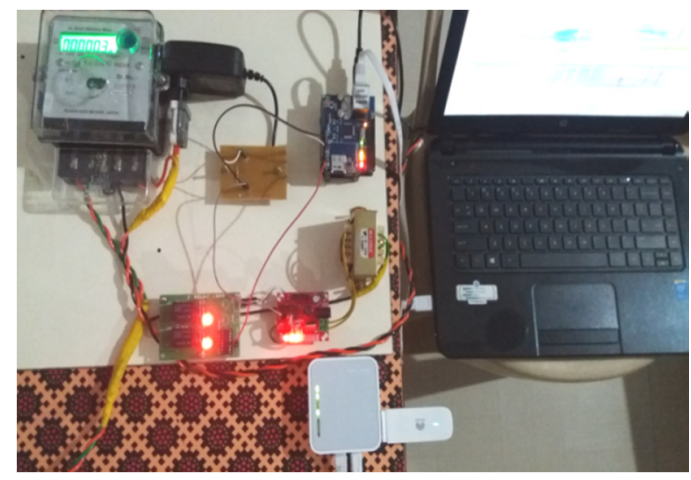

Fig. 3. Hardware implementation of the energy monitoring system using IoT

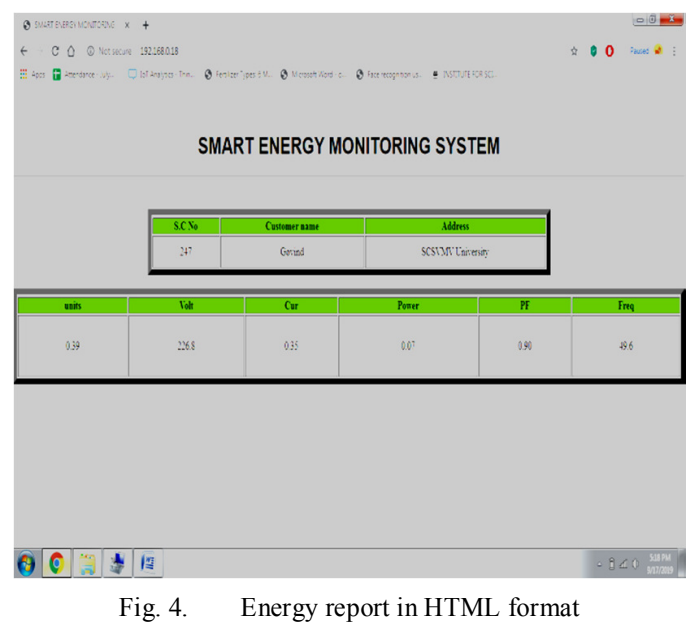

\section{Design and Implementation using Android Mobile App}

In this system, the control is using Android Mobile App (IEEE 1914.1) (a free and an open source framework for multiple platform mobile application development) developed as a part of User Interfaced (UI) MoSync Application program implemented on an android mobile phone. The user must have a mobile phone with internet or Wi-Fi access in most platforms except iOS. The application must be transferred and installed in the mobile phone. When the user launches the application, it displays the Initial screen shown in Figure 6. This screen has an option to exit the application or to connect to the embedded system which is interfaced with NodeMCU module. The user gets messages for the entire application activities comfortably. Once the application is installed in the mobile phone, it will automatically connect to the NodeMCU module using the static URL provided by the application itself. If the connection is successful then the client application gives an appropriate message and instructions to operate the system using the mobile device. Depending upon the type of the available input with the user's mobile device, the user can monitor power utilization.

In our designed system, Android Mobile App was utilized to develop a wireless energy monitoring system. It can sense the energy meter value and communicate with the consumer by showing energy information instantly [4]. It works with the android operating system and the module developed with 
NodeMCU, which is interfaced with our existing digital energy meter. The daily usage of power consumption will be measured as usual by a digital energy meter and the measured data will be received by the NodeMCU. Then the data will be sent to the existing mobile app, called Blynk which acts as a server. The Blynk App is one of the interfacing apps which can be installed on all android smart phones and it works with IoT based platforms. Once the data are received, they will be displayed as a prompt view on the mobile screen. The system obtains the energy parameters from the existing energy meter instantly, making them easily accessible with a mobile phone which is a user friendly device $[1,25]$. In other modules, more interfacing devices are utilized to develop an energy monitoring system, whereas in this system the number of interfacing devices is minimal, thereby reducing the data transferring speed considerably. The system consists of an energy meter, NodeMCU, and a smart phone. NodeMCU is a low-cost open source IoT platform. It runs on the ESP8266 Wi-Fi SoC and the hardware in which it is based on is the ESP-12 module.

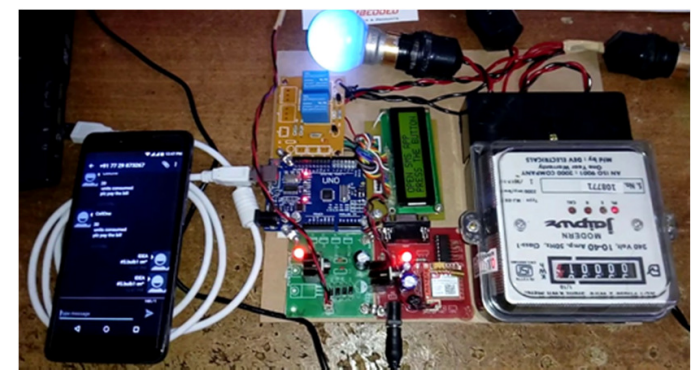

Fig. 5. Hardware implementation of the Android Mobile App-based energy monitoring system

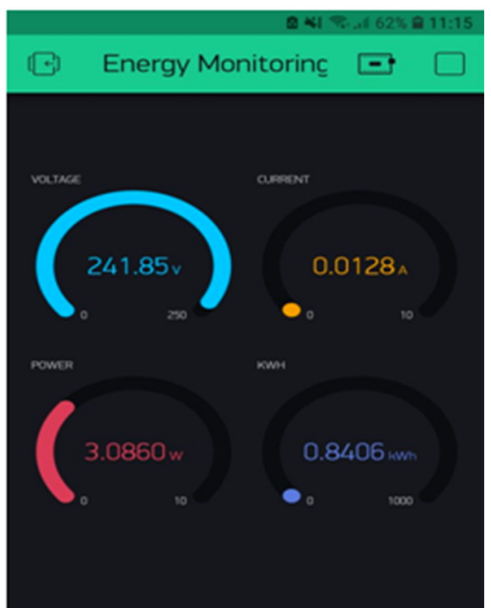

Fig. 6. Energy meter value displayed in mobile screen

Blynk is a customizable app, hence, based on the monitoring system requirements, the Blynk app page can be designed by the user. Once the app is installed in the mobile phone, the energy meter values will be stored in the mobile App by getting NodeMCU log in ID in the App. The developed Module is shown in Figure 5. At an interval of $1 \mathrm{~s}$, the parameters will be updated automatically. The output format of the energy monitoring system is shown in Figure 6.

\section{Design and Implementation using Cloud Computing}

Cloud computing is a most excellent and advanced method for smart energy monitoring due to its scalable and flexible characteristics and its capacity to handle large volumes of data. Its reliability, mobility, high speed, among other characteristics make it more adaptable than other methods. The design of a SEMS facilitates large-scale real-time computing capabilities in order to cope up with the communication, transport and storage of huge transferable data. But once the expected entities are in place, cloud computing will unload the smart system by offering automatic updates, remote data storage while demanding reduced maintenance of the IT systems, thus saving money, manpower, and energy. This system includes a two way digital energy meter and a GSM Communication Gateway (IEEE 802.21). The system is implemented in real time in order to achieve daily power utilization without delay. The consumer can check his energy usage as an instant report [21]. The system is designed with a smal number of interfacing devices to reduce development cost and data transferring time [22]. Here, the meter is directly connected with the GSM communication gateway and the measured parameters will be stored in our personal cloud. The GSM communication gateway is especially developed to collect the data from the meter and send them to the server hence it will act as the main processor. In order to get customized screens in the cloud server, programming is developed in super java. Based on the consumer requirements the measured energy parameters will be formed as a report which can be chosen for different time periods (daily, monthly or live). This prototype achieved cloud transmission level of 14Tbps without any delay [23].

The SATEC power meter is used to collect the data from the main panel to the consumer end. GSM Based Communication gateway circuit [24] was used. This system has been developed keeping in mind the aspects of reducing delay time for data transfer, increasing wireless coverage distance limit, and getting more values form the meter. In Zigbee based monitoring systems, the power metering IC and Arduino controllers were used and the system provided $15 \mathrm{~s}$ delay time in transferring data. In the IoT-based monitoring system, a splitter circuit, a data amplifier and the Arduino controller were used to collect the data and send them through the WiFi router. By IP address accessing mode we can view the data in an HTML file while the data are updated every $2 \mathrm{~s}$. In order to achieve fast communication with reliable data and more values from the meter, the wireless coverage limit is increased. In order to obtain the data storage unit, the cloud based smart energy monitoring is developed. Hence instead of power metering IC and splitter circuit we used the GSM communication gateway circuit directly interfaced with the smart meter as shown in Figure 7. The communication gateway will send the data to the server and the data will be saved as per our customized program in the cloud. The data can be accessed from anywhere and we can get a customized report without any difficulties. The transmission speed is $14 \mathrm{Tbps}$ without any delay time as the coding is developed for a fully online system. The system gives a customizable master report as shown in Figure 8 . The coding has been developed in super java for the communication unit and the measured values can be viewed as a report in a customized HTML file. 


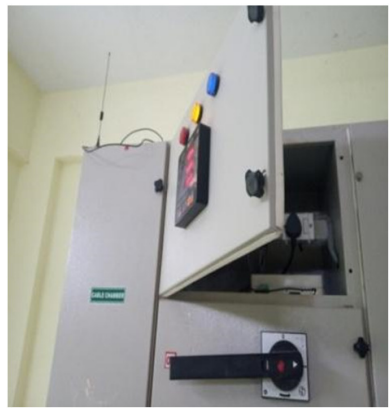

(a)

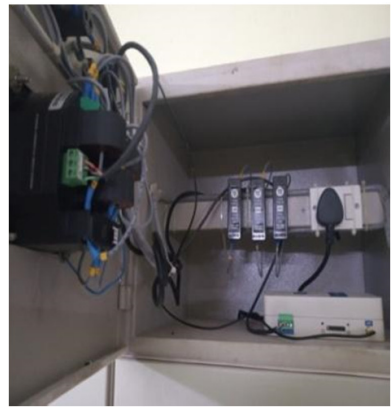

(b)
Fig. 7. Hardware installation of the energy monitoring system using the GSM gateway

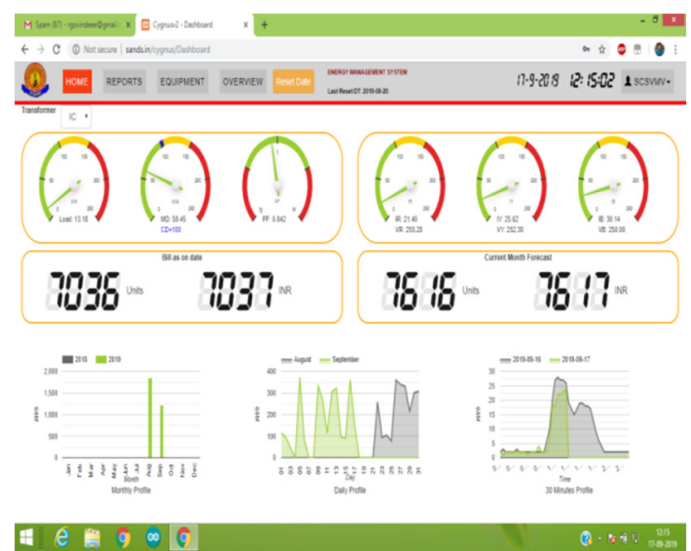

Fig. 8. Real time energy monitoring live report from the cloud

\section{PERFORMANCE ANALYSIS}

The implemented SEMSs with their real time measurements employing different communication modules are compared in Figures 9-10. The SEMS using GSM communication gateway and developed with cloud computing shown exhibits improved performance in LT consumers for daily utilization. This system provides detailed information to the LT consumers about their power consumption which includes live chart, day chart, and month wise chart. The overall comparison is shown in Table I.

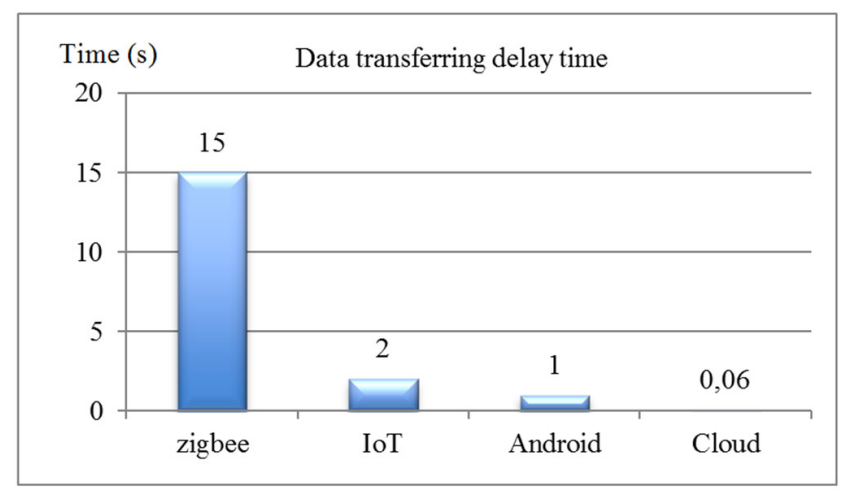

Fig. 9. Comparison chart of data transferring delay time in energy monitoring systems

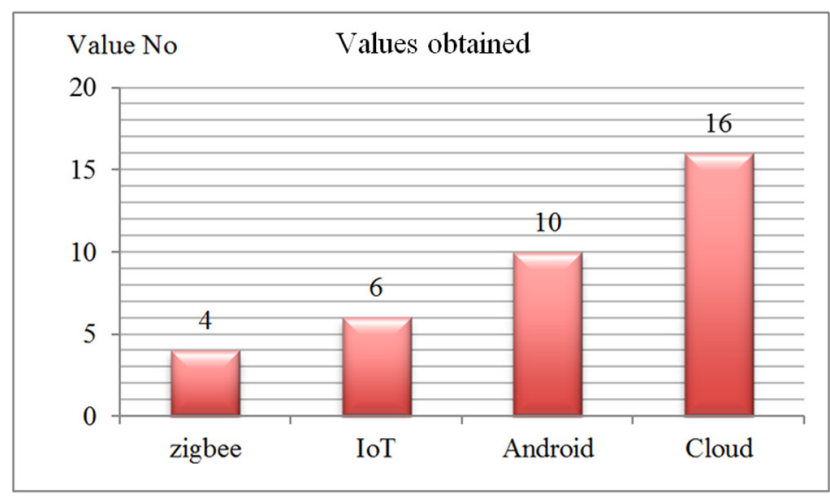

Fig. 10. Comparison chart of the number of values obtained by the energy monitoring systems

TABLE I. PERFORMANCE OF THE IMPLEMENTED SEMS

\begin{tabular}{|c|c|c|c|c|}
\hline System & Speed & Delay (s) & $\begin{array}{c}\text { No of } \\
\text { values }\end{array}$ & $\begin{array}{c}\text { Network coverage } \\
\text { limit }\end{array}$ \\
\hline ZigBee based & $250 \mathrm{kbps}$ & 15 & 4 & $100 \mathrm{~m}$ \\
\hline IoT based & $2 \mathrm{Mbps}$ & 2 & 6 & $92 \mathrm{~m}$ \\
\hline Android based & $424 \mathrm{kbps}$ & 1 & 10 & - \\
\hline Cloud based & $14 \mathrm{Tbps}$ & Real time & 16 & - \\
\hline
\end{tabular}

\section{CONCLUSION}

Different types of energy monitoring systems were developed and the performance of all aspects of the systems was analyzed and investigated by comparing the concept and technology used. The comparison of real time output results based on the communication module proves that the cloud computing-based smart energy monitoring system using GSM communication gateway produces improved performance in a number of parameters, namely high speed data communication, online data transfer, customized report making and in providing instant updated information. The cloud computing system practically responds in real time. With these systems, the consumers can know about their power utilities easily, making their power consumption schedule with this knowledge and calculate their electricity usage cost. Power consumption can be reduced by developing this kind of innovative model in real time.

\section{REFERENCES}

[1] J. Zheng, D. W. Gao, L. Lin, "Smart meters in smart grid: An overview", IEEE Green Technologies Conference, Denver, USA, April $4-5,2013$

[2] K. Vikram, S. Kumar, "Role of smart meters in smart grid", Electrical India, Vol. 57, No. 6, pp. 38-46 2017

[3] K. Baraka, M. Ghobril, S. Malek, R. Kanj, A. Kayssi, "Low cost arduino/android-based energy-efficient home automation system with smart task scheduling", $5^{\text {th }}$ International Conference on Computational Intelligence, Communication Systems, and Networks, Madrid, Spain, June 5-7, 2013

[4] M. M. Albu, M. Sanduleac, C. Stanescu, "Syncretic use of smart meters for power quality monitoring in emerging networks", IEEE Transactions on Smart Grid, Vol. 8, No. 1, pp. 485-492, 2017

[5] M. Ruth, A. Pratt, M. Lunacek, S. Mittal, H. Wu, W. Jones, "Effects of home energy management systems on distribution utilities and feeders under various market structures: Preprint", 23rd International Conference on Electricity Distribution, Lyon, France, June 15-18, 2015 
[6] R. Pereira, J. Figueiredo, R. Melicio, V. M. F. Mendes, J. Martins, J. C. Quadrado, "Consumer energy management system with integration of smart meters", Energy Reports, Vol. 1, pp. 22-29, 2015

[7] S. Hallur, R. R. Kulkarni, P. P. Patavardhan, "Smart components for a smart energy meter", International Journal of Advance Research in Engineering, Science \& Technology, Vol. 4, No. 3, pp. 544-556, 2017

[8] R. A. Rashid, L. Chin, M. A. Sarijari, R. Sudirman, T. Ide, "Machine learning for smart energy monitoring of home appliances using IoT", $11^{\text {th }}$ International Conference on Ubiquitous and Future Networks, Zagreb, Croatia, July 2-5, 2019

[9] T. S. Choi, K. R. Ko, S. C. Park, Y. S. Jang, Y. T. Yoon, S. K. Im, "Analysis of energy savings using smart metering system and IHD (inhome display)", Transmission \& Distribution Conference \& Exposition: Asia and Pacific, Seoul, South Korea, October 26-30, 2009

[10] W. H. Kim, S. Lee, J. Hwang, "Real-time energy monitoring and controlling system based on ZigBee sensor networks", Procedia Computer Science, Vol. 5, pp. 794-797, 2011

[11] W. H. Kim, C. H. Hyun, M. J. Kim, "Zigbee based wireless energy surveillance system for energy savings", International Journal of Electrical, Computers, Energetic, Electronic and Communication Engineering, Vol. 6, No. 10, pp. 1179-1183, 2012

[12] C. Prapasawad, K. Pornprasitpol, W. Pora, "Development of an automatic meter reading system based on ZigBee PRO smart energy profile IEEE 802.15.4 standard", IEEE International Conference on Electron Devices and Solid State Circuit, Bangkok, Thailand, December $3-5,2012$

[13] R. Govindarajan, S. Meikandasivam, D. Vijayakumar, "Energy monitoring system using Zigbee and Arduino", International Journal of Engineering \& Technology, Vol. 7, No. 4, pp. 608-611, 2018

[14] V. K. Sah, S. Brinda, J. Harish, U. Akshay, V. D. Mahto, S. Umapathy, "Smart energy meter", International Journal of Engineering Science and Computing, Vol. 8, No. 3, pp. 16578-16581, 2018

[15] A. S. Rao, S. V. Garige, "IoT based smart energy meter billing monitoring and controlling the loads", International Journal of Innovative Technology and Exploring Engineering, Vol. 8, No. 4S2, pp. 340-344, 2019

[16] B. Sahani, T. Ravi, A. Tamboli, R. Pisal, "IoT based smart energy meter", International Research Journal of Engineering and Technology, Vol. 4, No. 4, pp. 96-102, 2017

[17] A. Y. Devadhanishini, R. K. Malasri, N. Nandinipriya, V. Subashini, P. G. P. Gowri, "Smart power monitoring system using IoT", 5th International Conference on Advanced Computing \& Communication Systems, Coimbatore, India, March 15-16, 2019

[18] R. Govindarajan, S. Meikandasivam, D. Vijayakumar, "Low cost Arduino based smart energy monitoring system using internet of things", Journal of Engineering and Applied Sciences, Vol. 14, No. 1, pp. 170177,2019

[19] K. Chooruang, K. Meekul, "Design of an IoT energy monitoring system", Sixteenth International Conference on ICT and Knowledge Engineering, Bangkok, Thailand, November 21-23, 2018

[20] L. M. Satapathy, S. K. Bastia, N. Mohanty, "Arduino based home automation using Internet of things (IoT)", International Journal of Pure and Applied Mathematics, Vol. 118, No. 17, pp. 769-778, 2018

[21] B. Bhagyashree, S. Kulkarni, R. D. Patane, "Energy management technology based on cloud network", Journal of Electrical and Electronics Engineering, pp. 23-26, 2014

[22] N. D. Yadav, A. Wanjari, "Cloud based smart metering security access and monitoring system in the real time environment", International Journal of Engineering Research \& Technology, Vol. 3, No. 2, pp. 13491353, 2014

[23] R. Govindarajan, S. Meikandasivam, D. Vijayakumar, "Cloud computing based smart energy monitoring system", International Journal of Scientific and Technology Research, Vol. 8, No. 10, pp. 886-890, 2019

[24] P. Naveen, W. K. Ing, M. K. Danquah, A. S. Sidhu, A. Abu-Siada, “A cloud associated smart grid admin dashboard", Engineering, Technology \& Applied Science Research, Vol. 8, No. 1,pp. 2499-2507, 2018
[25] I. Androulidakis, G. Kandus, "Mobile phone brand categorization vs. users' security practices", Engineering, Technology \& Applied Science Research, Vol. 1, No. 2, pp. 30-35, 2011

\section{AUTHORS PROFILE}

R. Govindarajan was born in Tamilnadu, India, on 1982. He received his BE Degree in Electrical and Electronic Engineering from Sri Chandrasekharendra Saraswathi Viswa Mahavidyalaya, Kanchipuram, Tamilnadu, India, MTech. Degree in Applied Electronics from Bharath University, Chennai, Tamil $\mathrm{Nadu}$, India and he is currently pursuing his PhD in Electrical Engineering in SCSVMV University, Tamilnadu, India. He has published four research papers in Scopus indexed journals and two in other reputed journals. His areas of interest include smart gird, smart energy management, wireless communication, and cloud computing.

S. Meikandasivam received his BSc Degree in Electrical and Electronics Engineering from Anna University, Chennai, Tamilnadu, India in 2002, the MSc Degree in power systems in 2005, and a PhD Degree from the Maulana Azad National Institute of Technology, Bhopal, India in 2010. He has been an Associate Professor and Head of the School of Electrical Engineering, Vellore Institute of Technology, Tamilnadu, India.

D. Vijayakumar received his $\mathrm{BE}$ degree in Electrical and Electronic Engineering from Madras University, Chennai, India, in 2002, ME Degree in Power Systems from SCSVMV University, Tamil Nadu, India in 2005, and his $\mathrm{PhD}$ Degree in Electrical Engineering from Maulana Azad National Institute of Technology, Bhopal, India in 2010. He has been a professor with the School of Electrical Engineering, Vellore Institute of Technology, Tamilnadu, India. 